\title{
THE ULTRASTRUCTURE OF FLAGELLAR FIBRILS
}

\author{
DANIEL C. PEASE, Ph.D. \\ From the Department of Anatomy, School of Medicine, University of California at Los Angeles
}

\begin{abstract}
A BSTRACT
The tips of rat sperm tails were slightly frayed by mechanical agitation, thus exposing the fibrils, which were then studied by electron microscopy after negative staining. Only the fibrils survived this treatment. Each fibil proved to be a cylinder with a hollow core. The walls of the cylinders were made up of 10 longitudinally oriented filaments. The filaments had a markedly beaded appearance, with a repeating period of $88 \mathrm{~A}$. The filament thickness (bead width) was approximately 35 to $40 \mathrm{~A}$. Beads of neighboring filaments were in register with each other so that cross-linking bound the filaments together to complete the wall structure of each fibril. The center-to-center spacing from one filament to the next was 55 to $60 \mathrm{~A}$. The periodicity and the diameters of the filaments make it unlikely that the filaments are related to either actin or myosin. From the way the fibrils kinked, it can be inferred that they possessed considerable mechanical strength. It is consistent with present knowledge that fibrils of the mitotic apparatus may have the same basic structure as the flagellar fibrils. Under some circumstances, pairs of fibrils separated from one another along their length, except at their extreme tips. It was apparent that there was special bridging material to be found there. In other preparations, however, the paired fibrils remained together, indicating a powerful coupling mechanism.
\end{abstract}

Electron microscopy obviously is necessary to reveal the ultrastructure of cilia and flagella. They have attracted the attention of electron microscopists since the early years of biological applications. It seemed evident from the first that ordered structures would be found that perhaps could be correlated with other systems which produce mechanical movement. Thus, there was reasonable hope of making scientific progress easily and quickly. The latter thought has proved to be a will-o'-the-wisp, although simple fragmentation procedures, coupled with shadowcasting, soon did reveal the presence of fibrils within flagella (Grigg and Hodge, 1949, and Manton and Clarke, 1952).

Ultrathin sectioning techniques had to be substantially developed before further progress could be reported. In a now classical paper, Fawcett and Porter, 1954, recognized that the fibrils almost invariably appeared as a ring of 9 pairs, with two more single ones in the center. In subsequent years numerous investigators have demonstrated the near universality of this pattern. This literature is reviewed by Fawcett, 1961.

Technical advances in microscope design and microscopy during very recent years have encouraged further studies with improved preservation and greater resolution. This has led to the publication of important papers by Afzelius, 1959, 1961; André, 1961; Gibbons and Grimstone, 1960; and Gibbons, 1961. These several workers are in remarkable agreement with each other in spite of working with very diverse material. They have demonstrated much patterned structure within cilia and flagella aside from just the presence of the fibrils. This work, however, has depended entirely upon the ultrathin sectioning of fixed preparations.

The advent of negative staining techniques, particularly fostered by Huxley, 1956, and by 
Horne and Brenner, 1958, and Brenner and Horne, 1959, has provided an important new tool for the study of isolated cell particulates and macromolecules. Thus, there has been a revival of interest in fragmenting and dispersing cellular components. It is logical to explore the potentialities of these new developments in relation to the structure of cilia and flagella. The present investigation was undertaken with this in mind, and it has proved possible to see orders of structure within the flagellar fibrils that have not been observed at all with other methods of observation. It is evident that one can indeed visualize an organized macromolecular pattern of the units making up these structures. Although the method surely can and will be refined beyond what is presented in this paper, it is felt that enough new information of sufficient importance has been obtained to warrant publication at the present time. ${ }^{1}$

In addition to demonstrating structural components within flagellar filaments at high resolution, it also has been possible to deduce some new information concerning the physical properties of the fibrils from the patterns observed when these have been isolated.

1 The reviewing editors of The Journal of Cell Biology have indicated to this author that micrographs somewhat comparable to those presented here were shown by Dr. I. R. Gibbons at the Fifth International Congress for Electron Microscopy. However, the published abstract of his paper (Electron Microscopy, New York, Academic Press, Inc., 1962, 2, M-2) makes no reference to them and this writer did not hear the talk. The present work was performed entirely independently and without knowledge of the results obtained by Dr. Gibbons.

\section{MATERIALS AND METHODS}

Rat spermatozoa, removed from the epididymis, provided the material for this investigation. Concentrated sperm were removed "dry" from the distal end by making small cuts through coiled portions of the duct upon the exposed testes of narcotized animals. Bleeding usually was minimized by clamping the spermatic cord just before doing this. When concentrated sperm had oozed out of a fresh cut through the epididymis, they were picked up with a toothpick and transferred to a small beaker containing 1 to $2 \mathrm{ml}$ of Na-phosphotungstate or, in some instances, first to 10 per cent buffered formalin. The sperm were dispersed in these media by shaking, and scraping with a second toothpick. After several samples had been collected, an effort was made to fragment the sperm tails by violent agitation. This was accomplished by the repeated sucking of the suspension through a fine bore hypodermic needle, and then squirting it back into the container. In spite of the mechanical and osmotic violence of the treatment, most flagella remained intact along most of their lengths, excepting that the extreme tips often were ruptured (Fig. 1). The tip is morphologically distinct from the rest of the flagellum in that it lacks the "fibrous sheath" of the rest of the shaft. It seems evident that the fibrous sheath, in fact, offers important structural support to much of the sperm flagellum, and it is only the extreme tip that is particularly fragile.

The dispersion medium in most instances was 0.5 to 2 per cent Na-phosphotungstate, prepared by titrating phosphotungstic acid with concentrated sodium hydroxide until the $\mathrm{pH}$ reached the desired level (it was deliberately varied between 6 and 7.4). When the dispersion medium was 10 per cent formalin in a phosphate buffer, a short fixation of about 10 minutes was followed by the addition of sufficient Na-phosphotungstate to yield a final concentration

FIGURE 1 A disrupted sperm tail tip showing "clean" fibrils spread out over the supportfilm. In general, pairs of fibrils have remained coupled together as the divergent ends of the one marked 15,16 indicate. In these instances one fibril usually is on top of the other and thus is not clearly seen. The tips of most fibrils have been flattened, and are ribbonlike. Details of two of these fibrils are repeated in subsequent figures as indicated by those marked 11,12 and 15,16 . In this specimen it is evident at the base of the figure that 9 coupled pairs of fibrils can be counted. Additionally, 2 other fibrils are but loosely associated. The latter extend vertically to the top margin of the picture, and no doubt represent the "central fibrils" of the flagellum. A portion of one of these is shown at high magnification in Fig. 14. Micron marks accompany this and subsequent figures. $\times 44,500$.

FigURE 2 Fibrils which have been kinked by excessive mechanical force. Three bends are indicated by the arrows. In this instance the fibrils have remained paired, and somewhat "ghostly" images of the superior fibrils are seen overlaying the dominant images of the inferior ones. $\times 148,000$. 

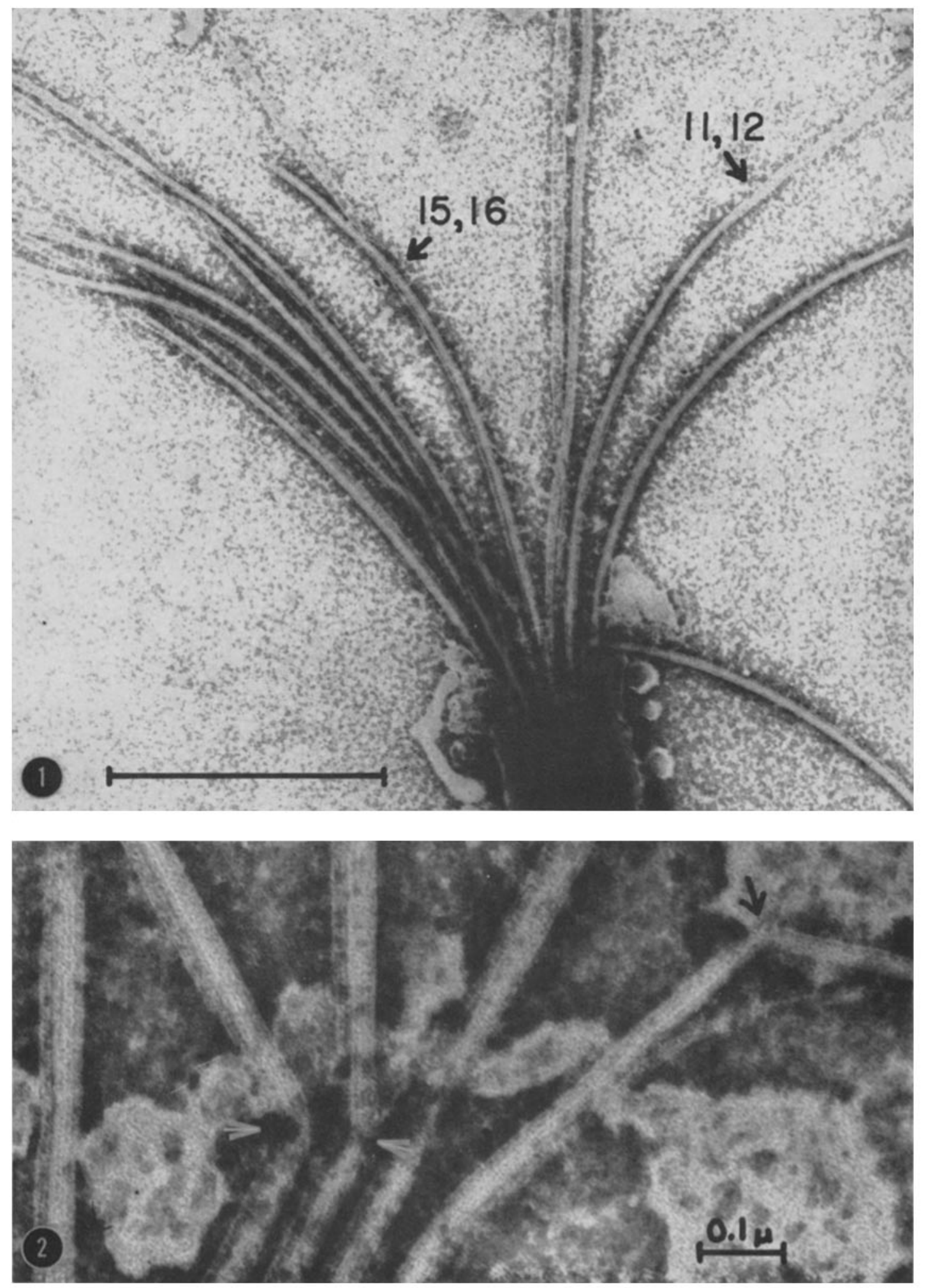

Daniel C. Pease Ultrastructure of Flagellar Fibrils 
of the latter of I per cent. The latter method undoubtedly is potentially useful, but its possibilities were not extensively explored in the present investigation. It was found harder to fragment such "fixed"، sperm than living cells added directly to the staining solution.

Various methods of preparing the specimen grids were tried. These included "nebulizing" and spraying the suspension upon grids previously coated with Parlodion films reinforced with evaporated carbon. The simplest method, however, yielded by far the best and most consistent results. This involved only the placing of a drop of suspension on a coated grid, and waiting for a few minutes before removing most of the drop with a bit of filter paper. The residual fluid was allowed to dry down upon the specimen, leaving its deposit around the dried sperm. Inevitably the technique was hard to standardize completely, and the results were quite variable. One parameter, which was probably of considerable significance, was the amount of serum and other protein that was included with the suspension. In some instances the residual stain deposit was nearly amorphous, even at high magnification. In other instances, particulate deposits remained. This most likely can be attributed to the relative presence or absence of protein colloid, which in turn affected the wettability of the carbon-coated surface of the supporting film. In this group of experiments, which were fundamentally of an exploratory nature, the variation was welcomed and gave useful information, although the exact mechanics of what happened during specimen preparation were not fully understoöd.

The most informative micrographs actually came from two basic preparations, and are used for all but one of the illustrations of this article. Figures 3 to 9 are from a sample suspended in incompletely neutralized phosphotungstic acid which was contaminated with a good deal of serum. On the other hand, Figs. 1 and 10 to 18 are from a "clean" preparation suspended in $\mathrm{Na}$-phosphotungstate buffered at $\mathrm{pH}$ 7.4. As will become apparent, the patterns visualized gave complementary information.

All critical measurements were based upon calibrations of the microscope at the time of making the original micrographs. This was done by micrographing a replica of a diffraction grating at appropriate magnifications.

\section{RES ULTS}

When flagellar tips were disrupted as described in the previous section, the only structural elements which survived were the 9 pairs of peripheral fibrils, plus the unpaired central ones. It is possible and even likely that if more sophisticated methods of fragmentation had been employed, following more elaborate fixation, additional structures might have been seen. The dissolution of all other components served one valuable purpose, however, in that the fibrils could be observed in a "clean" condition. It was then apparent that their basic structure was that of a cylinder, the wall of which consisted of a small number of filaments. (In what follows, a sharp terminological distinction is made between "fibrils," the conventionally known structures, and "filaments," their substructures.)

Fig. 1 shows how the flagellar tips commonly were disrupted, exposing the internal fibrils. At high magnification, and under suitable conditions of staining, individual fibrils could be seen to consist of longitudinally oriented filamentous components (Figs. 11 to 18). In uncollapsed fibrils, either 5 or 6 filaments ordinarily could be seen (Figs. 11, 13, 14, and 18). The filaments sometimes were obviously beaded (Figs. 11 to 18 ), with a repeating period of about $88 \mathrm{~A}$, as will be described later. The width of individual beads could not be measured with great accuracy, but was approximately 35 to $40 \mathrm{~A}$. A more accurate, and perhaps more significant measurement, was the center-to-center spacing of adjacent filaments, for these were arrayed in regular order (Figs. 11 to 18). This spacing was between 55 and $60 \mathrm{~A}$.

There were two lines of evidence that the flagellar fibrils were hollow structures, having as their walls a small number of the linearly oriented filaments. First, in some preparations, the hollow centers had been filled with the Na-phosphotungstate, leaving only the vertically oriented portions of their wall structures unstained and clearly visible (Figs. 3 to 6 ). These micrographs are incompatible with any supposition that there

Figures 3 throvgh 6 Fibrillar tips from a preparation different from that represented by Fig. 1. In this instance, the pairs of fibrils have tended to separate, except at the extreme tips where bridges of amorphous material have continued to exist. Note that the cores of these fibrils have been filled with Na-phosphotungstate, and it is only the side walls that remain clearly visible. $\times 71,000$. 

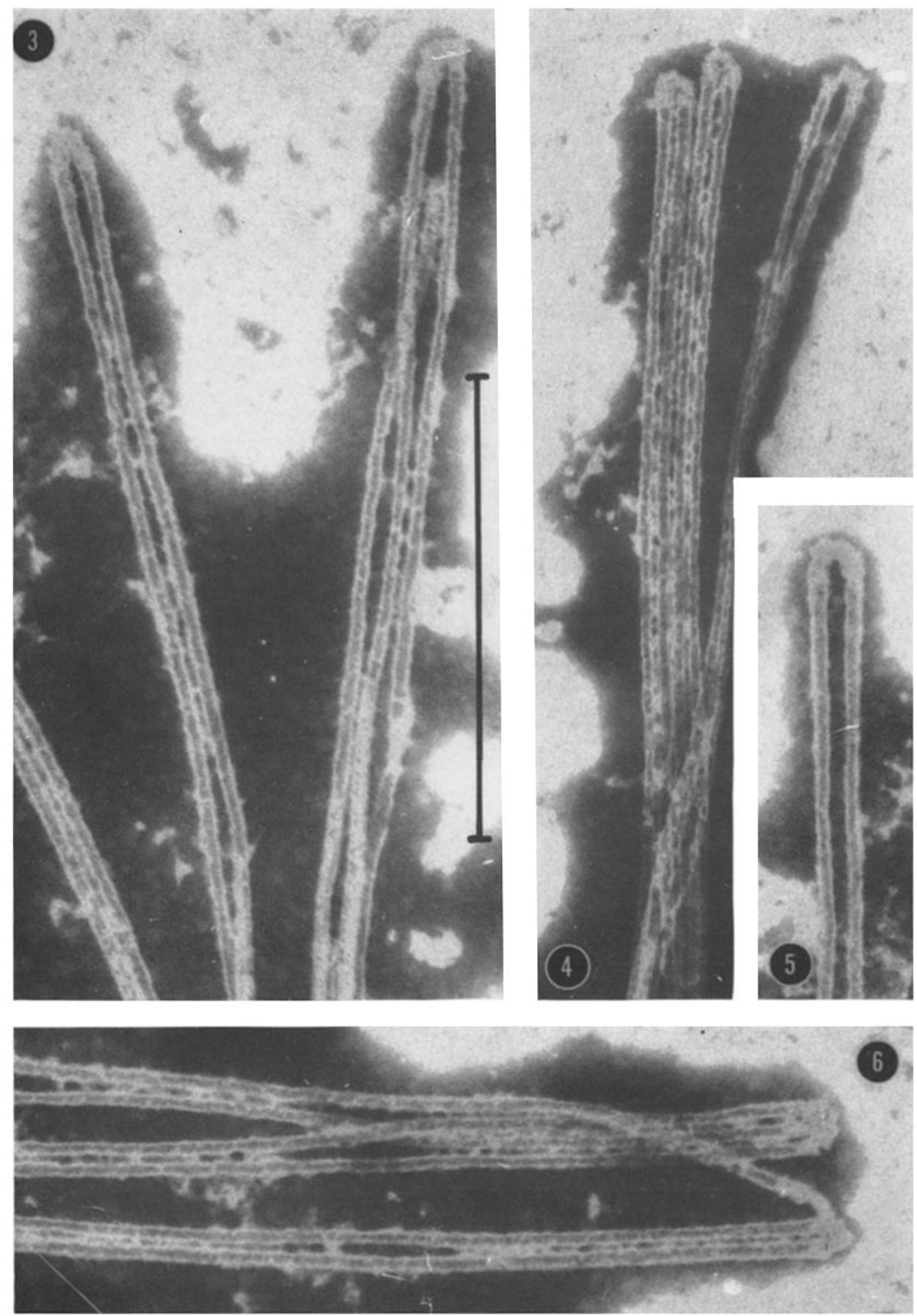

Danigt, C. Panse Ultrastructure of Flagellar Fibrils 
might be a cylindrical solid bundle of filaments. Second, in other preparations, it was commonplace to observe that fibrils became abruptly flattened and ribbon-like (Figs. 10 to 12, also Fig. 1). So long as the fibrils remained cylindrical, there was a tendency for the Na-phosphotungstate to pile up on their sides as conspicuous dense bands. When flattening occurred, there was concomitant widening, and not the accumulation of the staining material along the edges. Also, wherever the tubular structure had collapsed, it was ordinarily possible to observe more than 6 filaments. Indeed, 10 filaments seemed to be the maximum number, and probably the constant number, in each flagellar fibril.

Theoretical and geometrical reasons make it evident why one would expect to see only 5 to 6 filaments in an intace cylindrical wall structure consisting of 10 filaments. Under the conditions of observation, only those filaments close to or in contact with the supporting film would be outlined by the Na-phosphotungstate. The uppermost filaments would not be seen. The observed accumulation of Na-phosphotungstate on the sides of uncollapsed fibrils indicated that just about half of each fibril was stained, including that part in contact with the supporting film. Thus, either 5 to 6 or 10 filaments were seen, depending upon whether an intact cylinder was observed, or a cylinder which had been split, unfolded, and flattened as a ribbon.

It has been established from transverse sections that the two fibrils of a coupled pair are not identical, one member usually having asymmetric side arms, (Afzelius, 1959, and Gibbons and Grimstone, 1960). In the present work, arms have not been identified, and it has not been possible to distinguish differences between the two fibrils of a pair. As is evident in Fig. 10, both fibrils of a pair might be similarly split, and expanded as ribbons. Also, the "central" fibrils had the same fundamental filamentous structure as the "peripheral" ones (Figs. 1, 13, and 14).

The periodic beading of the filaments was seen to be in register from one filament to the neighboring ones, thus indicating definite cross-linkages. This is particularly evident in places in Figs. 13 and 14. In this connection it should be noted further that even when a fibril was split and flattened, there was no tendency for individual filaments to separate. Rather, they stayed bound together laterally, forming a ribbon (Figs. 1 and 10 to 12).

A different preparation, which did not show individual filaments, did show particularly well a marked cross-banding, examples of which are illustrated in Figs. 7 to 9. In this instance, fortunate circumstances apparently exaggerated the pattern of the filament beads which were transversely in register. It was in this preparation that many consecutive periods could be counted and measured, so that the interperiod spacing could be determined quite accurately. In this particular preparation the spacing was found to average $88 \mathrm{~A}$, which is slightly higher than the 80 to $85 \mathrm{~A}$ spacings measured less accurately in other preparations. If the fundamental filamentous structure of the fibrils had not been seen in other preparations, however, it would not have been possible properly to interpret the cross-banding of this specimen.

The transverse banding evident in the preparation represented by Figs. 7 to 9 showed some evidence of obliquity. Thus it may be that neighboring filaments are not coupled exactly at right angles to their own axes, but rather, a helical arrangement may obtain.

It may be deduced that the flagellar fibrils have a substantial structural rigidity. In spite of having been nakedly exposed, they invariably tended to be either straight or only gently curved (Figs. 1, 3 to 10). Thus, they were not limp when preserved, even though denuded of other possible supporting structures. One obtains further insight from the manner in which they abruptly kinked when they had been forced to bend excessively. Fig. 2 shows an area where three fibrils had been so damaged. It is evident that they had buckled at very localized places, while most of each fibril retained its proper form. One observes exactly the type of buckling that would obtain from bending a thin walled metal tube to the point where the wall structure collapsed locally. Also, it was observed that if fibrils had been broken, this had happened abruptly with-

Frgures 7 to 9 Fibrils from the same preparation as that of Figs. 3 to 6 , in this instance showing in places a regular transverse banding. The repeating period of the fibrils is $88 \mathrm{~A}$.

In Figs. 7 and 8 the bridged tips of paired fibrils are evident. $\times 143,000$. 

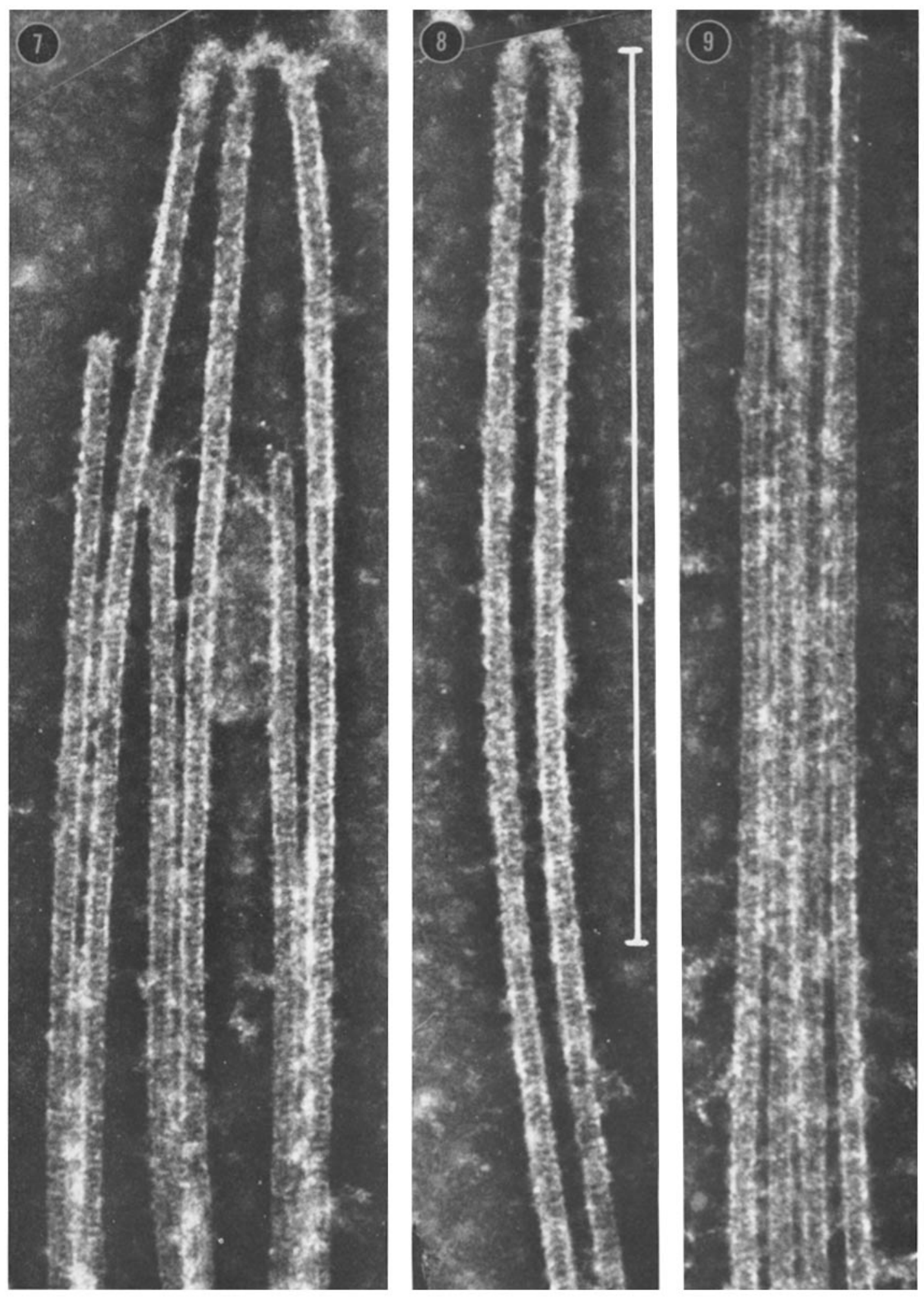

Daniel C. Pease Ultrastructure of Flagellar Fibrils 
out evidence of stretch and without involving neighboring regions.

Negative staining of frayed flagellar fibrils also has revealed a curious morphological relationship without evident significance. At the extreme tips of many pairs of fibrils in one particular preparation there was observed a small cloud of material that seemed essentially amorphous (Figs. 3 to 8 ). This material bridged across from one fibril to the other in spite of the fibrils' being otherwise separated. In some instances two or more pairs were all welded together in this manner (Figs. 6, 7). It apparently made a reasonably firm connection between the paired fibrils which survived under conditions which otherwise allowed filament pairs to break apart. These terminal connections between pairs of fibrils included even the two central ones for, in one instance, it has proved possible to count ten pairs so connected in a single flagellum. It is possible that the terminal connections were frayed bits of the original plasma membrane, but this is uncertain.

In some preparations it was evident that pairs of flagellar fibrils had remained rather firmly adherent one to the other (Fig. 1). In other preparations they had separated except, sometimes, for the terminal connections described above (Figs. 3 to 9). If one considers the poorly standardized conditions of specimen preparation, this variation does not seem surprising. It is possible that $\mathrm{pH}$ differences alone might have been responsible for the differences, although perhaps more subtle forces were at work. In any case, the bond between paired fibrils was a substantial one that could survive much traumatic abuse, although separation occurred before individual fibrils were seriously disrupted.

\section{CONCLUSION AND DISCUSSION}

As the result of this investigation it is concluded that flagellar fibrils are hollow structures. In the flagella of rat sperm, the wall of each cylindrical fibril consists of (probably) ten beaded filaments, longitudinally oriented and having a repeating period of about $88 \mathrm{~A}^{2}{ }^{2}$ The beads of neighboring filaments are in register with each other. In the past, it has not been possible to interpret sectioned or shadowed material in this way. Yet the present interpretation is not in conflict with what has been observed. Recent investigators, including Afzelius, 1959, 1961; Gibbons and Grimstone, 1960; Gibbons, 1961; and André, 1961; all have observed a dense ring structure in transverse sections of fibrils fixed with osmium tetroxide, as did Fawcett and Porter, (1954), in their now classic study of ciliary structure. This, of course, is in no way inconsistent with the concept of the fibril's being a hollow cylinder as described in the present article. It simply means that the filamentous nature of the cylinder walls had not been demonstrated by the more conventional fixation and staining procedures.

Periodicity specifically related to the fibrils has not been demonstrated convincingly by conventional techniques either, although a periodicity of much more than $88 \mathrm{~A}$ has been observed in the central region of flagella and cilia, and Gibbons and Grimstone, 1960, observed an apparent cross-striation of the central fibrils, with a periodicity of about $130 \mathrm{~A}$. This seemed to be obliquely arranged and they suggested a fundamentally helical pattern. Slight traces of a similar pattern also were observed in the peripheral fibers, although not clearly demonstrated. A similar

${ }^{2}$ Just before the publisher's proof of this paper was received, J. André and J.-P. Thiéry published a report of very similar experiments, (J. Micr., 1963, $2,71)$. Their results are in substantial agreement with mine in demonstrating 10 or 11 filaments, about $35 \mathrm{~A}$ wide, with an axial periodicity of approximately $80 \mathrm{~A}$, arranged as the tubular walls of the fibrils.

Frgure 10 A group of fibrils from the same preparation as that of Fig. 1. To the right of the figure, most of the fibrils have retained their cylindrical form with much Na-phosphotungstate piled up at their edges. To the left of the figure, most of the cylinders have collapsed, producing ribbons of filaments. The ribbons are wider than the cylinders, and lack the concentration of Na-phosphotungstate along their edges. Arrows indicate where this change in geometry can be seen to advantage. In this instance it was possible to identify the uppermost pair of fibrils as the "central" pair. This was the only uncoupled pair of the full complement of 10 visible in the vicinity of the surviving part of the flagellar shaft. $\times 133,000$. 


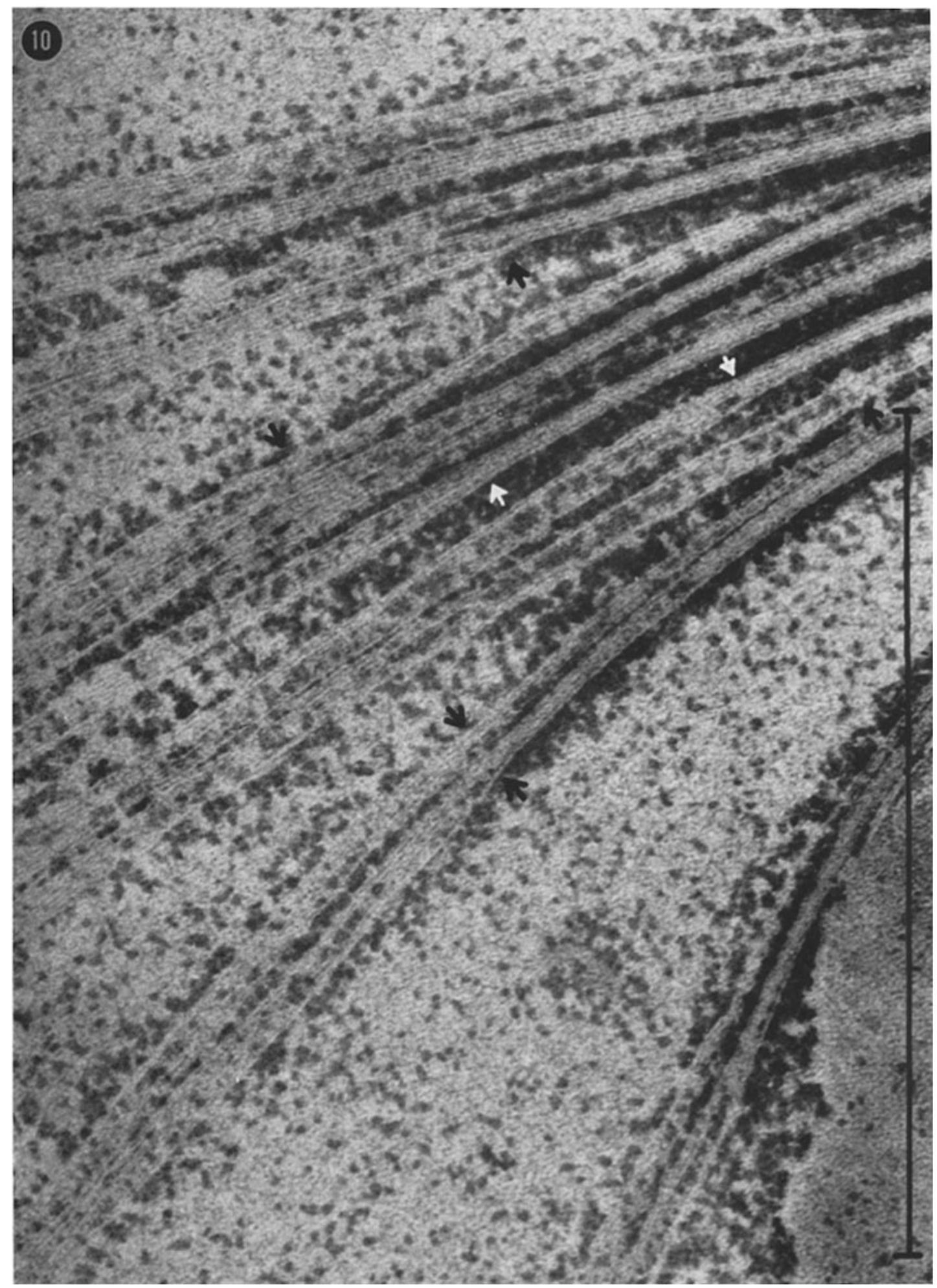

Daniel C. Pease Ultrastructure of Flagellar Fibrils 
periodicity also was seen in the interspaces between fibers in the central region. André, 1961. apparently observed the same repeating structure, and measured the periodicity as $120 \mathrm{~A}$. It seems impossible to reconcile these figures with the $88 \mathrm{~A}$ periodicity observed in the present work, even by assuming a simple multiple. It is quite possible, however, that the previous investigators were observing a repeating pattern in interfilamentous spaces. Thus, both groups of investigators speak of and emphasize an ill defined "central sheath" which is a possible locus of their pattern. Thus, the apparent inconsistency of their work with that presently described is most easily resolved by assuming that totally different structures were being visualized. We must adopt the view that the techniques of sectioning fixed and stained material, and the negative staining of isolated parts, complement each other, and so may extend knowledge, without necessarily duplicating it.

There has been, of course, a continuing temptation to try to interpret flagellar and ciliary movement on the basis of the generally better understood mechanisms of muscle contraction. Although this has led to much speculation, it has not proved to be particularly fruitful, and the findings of the present investigation raise new obstacles, although, of course, this is tempered by new information which eventually may prove useful.

The flagellar fibrils have an unexpected pattern that, at first thought, seems not to be correlated with that of other structures involved in mechanical movements. The dimensions of the unit filaments do not have very exact known parallels elsewhere. Thus, Huxley, 1957, indicates that the "primary" (myosin) filaments of skeletal muscle have a diameter of 110 to $120 \mathrm{~A}$, the "secondary" ones (actin), 50 to $60 \mathrm{~A}$. Thus, even the latter are substantially thicker than the filaments of the flagellar fibrils as measured directly (35 to $40 \mathrm{~A}$, although the center-to-center spacing of neighboring filaments indicates an "effective width" of 55 to $60 \mathrm{~A}$ ). In speaking of vertebrate smooth muscles, Schoenberg, 1962, comments that the filaments range from 50 to 80 $A$ in diameter. This makes them substantially thicker than what has been observed in the present investigation.

The fine filaments of striated muscle, and probably at least some of those of smooth muscle, are now generally thought to be actin (Hanson and Lowy, 1962). It has been possible to study such filaments in isclation by negative staining techniques. Huxley, 1962, as well as Hanson and Lowy, 1962, have been able to demonstrate periodicity along these filaments. The former investigator reported a 50 to $55 \mathrm{~A}$ spacing, the latter group of investigators found a mean of 56.5 A. The latter investigators also strongly suggested that each "nodule" represented a monomer of actin with an active site for combination with myosin at approximately every $55 \mathrm{~A}$ along each strand. These dimensions simply do not correspond with those observed in the flagellar filaments. It should be noted also that Huxley found that isolated actin filaments are 60 to $70 \mathrm{~A}$ thick, while Hanson and Lowy observed a diameter of $55 \mathrm{~A}$. Thus it seems most unlikely that the flagellar filaments are actin unless the monomers are arranged altogether differently than in muscle. Nor does it seem possible that they are myosin.

Although there is doubt about whether or not bacterial flagella are motor organelles, any homologies that could be established with the cilia and flagella of more complex cells would be particularly valuable, for it has proved possible to isolate and characterize the protein of the former. This literature has been reviewed by Kerridge, Horne, and Glauert, 1962, who have

Figures 11 and 12 Overlapping micrographs of a single flagellar fibril through the zone where the cylindrical form has collapsed, and a flat ribbon of isolated filaments has spread out. Five filaments are evident in the cylindrical region (Fig. 11), and ten in the expanded portion (Fig. 12) ( $x-x$ and $y-y$ represent similar lines on the two figures, and demark the zone of overlap). (Fig. 1 is a survey picture of the particular sperm tail from which this fibril was selected, as indicated by 11,12 there.) $\times 222,000$.

Figures 13 and 14 Portions of uncollapsed flagellar fibrils, both showing 6 beaded longitudinal filaments. In numerous places it can be observed that the beads of neighboring filaments are in register. Fig. 14 is of a "central fibril" as can le observed in the survey micrograph, Fig. 1. Likewise, Fig. 13 is of a "central fibril, one of the uppermost pair to be seen in Fig. 10. $\times 222,000$. 

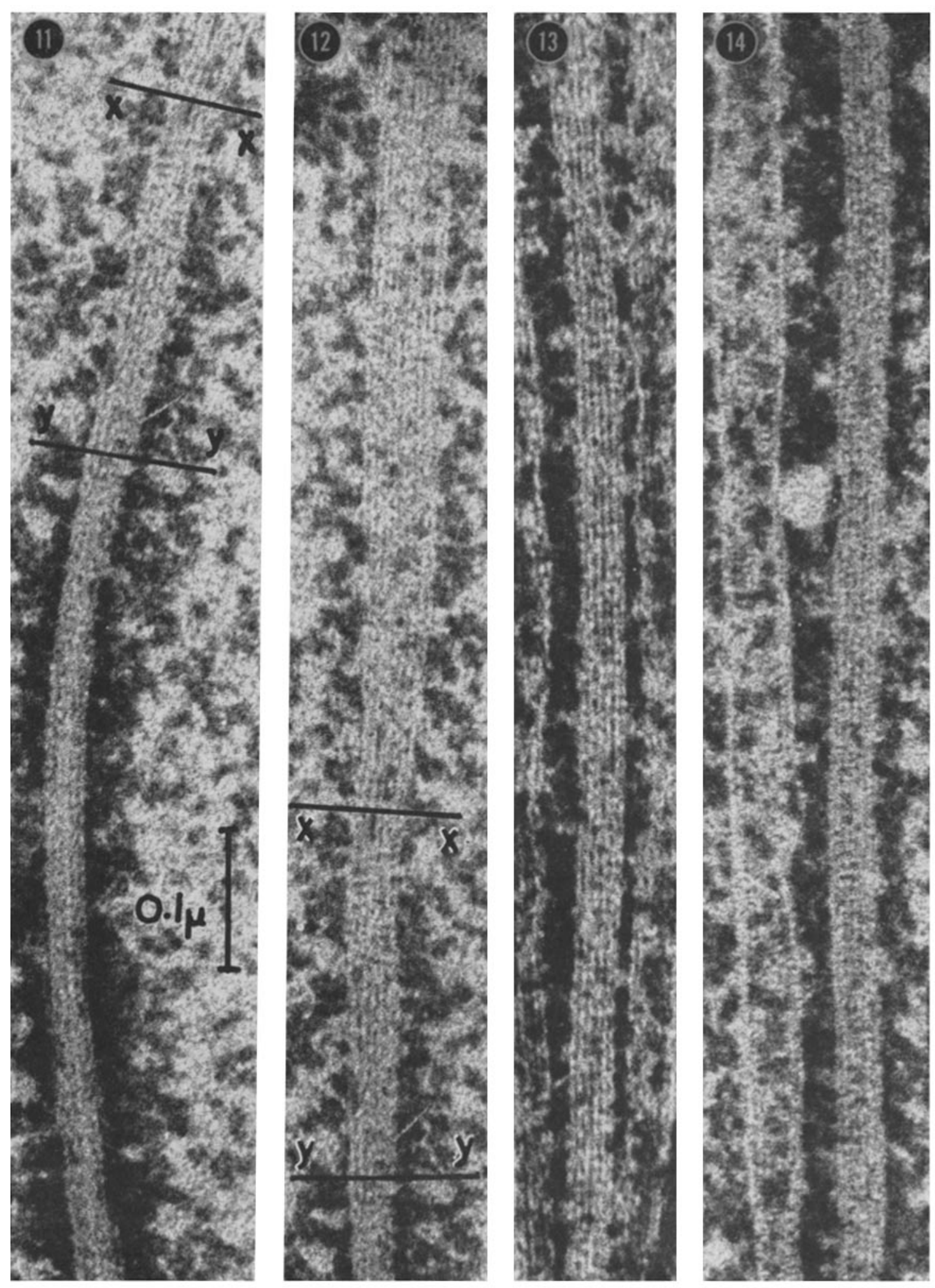

Daniel C. Pease Vltrastructure of Flagellar Fibrils 
added important information on the ultrastructure of partly degraded flagella from Salmonella. It is evident that the complex morphology found in the cilia and flagella of higher plant and animal cells is absent in the bacterial structures. Indeed, the latter can be compared only with the unit fibrils of the former. In the bacterial fibril Kerridge et al. observed hexagonally packed "beads," about $30 \mathrm{~A}$ in diameter, and $45 \mathrm{~A}$ center-to-center. Short linear strands sometimes could be observed, and pentagonal profiles were seen in transverse sections. These facts led them to propose three possible interpretive models, two of which involved helical coiling of 1 or 3 filaments, and the third, a parallel arrangement of 5 strands. Dr. R. J. Martinez has shown this writer unpublished micrographs of the flagella of Bacillis subtilis which clearly demonstrate a small number of beaded filaments in parallel array. This may correspond with the third model of Kerridge et al., although this was not their preferred arrangement. It would, however, agree better with present findings. The "bead" width, and particularly the spacing, reported by Kerridge and his co-workers seem significantly less than what is presently described, and no linear periodicity approaching the $88 \mathrm{~A}$ reported here was observed. Thus the evidence at hand is indecisive as to whether or not bacterial flagella may be homologous with the fibrils of the cilia

\section{LITERATURE CITED}

Afzelius, B., J. Biophysic. and Biochem. Cytol., 1959, 5, 269.

Afzelius, B., J. Biophysic. and Biochem. Cytol., 1961, 9, 383.

ANDRÉ, J. J. Ultrastruct. Research, 1961, 5, 86.

Brenner, S., and Horne, R. W., Biochim. et Biophysica Acta, 1959, 34, 103. and flagella of complex cells. Clearly there are some discrepancies, but these may not prove meaningful, for an organizational pattern based upon 5 or fewer filaments might be quite different from one utilizing 10 .

The relationship between "basal body" and flagellum, on the one hand, and centriole and mitotic spindle fibrils, on the other, is well known. It eventually may prove fruitful to compare the structure of flagellar fibrils with the mitotic ones. It is only recently that preservation techniques apparently have advanced to the point where satisfactory micrographs of sectioned mitotic fibrils have become possible (Harris, 1961, and Roth and Jenkins, 1962). It is apparent that the mitotic fibrils have osmiophilic rims and low density centers. They might be interpreted as cylindrical structures, just as are the flagellar fibrils. They are also of the same order of thickness as the flagellar fibrils. It is to be hoped that these fibrils can be isolated and studied with negative staining procedures. It is consistent with the evidence at hand that the mitotic fibrils may have basically the same structure as the flagellar ones. Thus, an understanding of the functional mechanics of one system may help importantly in understanding the other.

This study was supported in part by grants of the United States Public Health Service.

Receivcd for publication, January 7, 1963.
Fawcetr, D. W., Cilia and flagella, in The Cell, (J. Brachet and A. E. Mirsky, editors), New York, Academic Press, 1961, 2, Chapter 4, 217.

Fawcett, D. W., and Porter, K. R., J. Morphol., $1954,94,221$.

Gibsons, I. R., J. Biophysic. and Biochem. Cytol., 1961, 11,179 .

Frgures 15 то 18 Beaded longitudinal filaments are evident in these micrographs of flagellar fibrils. The diameter of the beads is approximately 35 to $40 \mathrm{~A}$, and the center-tocenter spacing is 55 to $60 \mathrm{~A} . \times 290,000$.

Figures 15 And 16 Overlapping micrographs of a single pair of fibrils, $x-x$ and $y-y$ being comparable lines on both figures demarking the areas of overlap. (Two superimposed fibrils actually are seen here, as the survey picture, Fig, 1, indicates, in which one can see the separate fibrils diverging.)

Frgures 16 and 17 Two different focal levels of the same fibril.

FIGURE 18 A fibril in which 6 filaments (and a marginal trace of a 7th) are visible. 

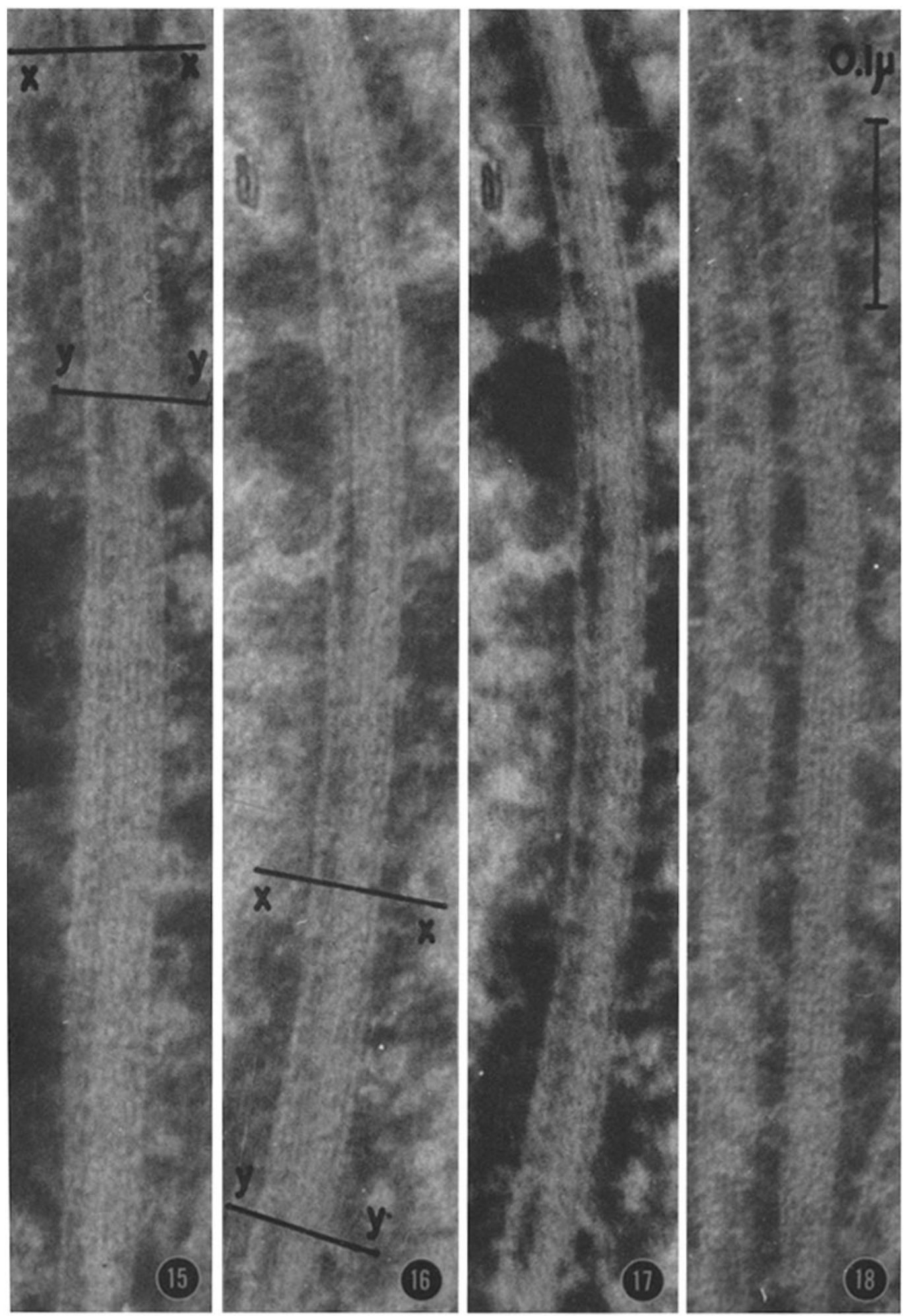

Danigi, C. Pease Ultrastructure of Flagellar Fibrils 
Gibbons, I. R., and Grimstone, A. V., J. Biophysic. and Biochem. Cytol., 1960, 7, 697.

Grigg, G. W., and Hodge, A. J., Australian J. Sc. Research Series B, 1949, 2, 271.

Hanson, J. and Lowy, J., Electron Microscopy, (Proc. 5th Internat. Congr. Electron Micr., Philadelphia), New York, Academic Press, 1962, 2, $0-9$.

Harris, P., J. Biophysic. and Biochem. Cytol., 1961, $11,419$.

Horne, R. W., and Brenner, S., 4th Internat. Congr. Electron Micr., Berlin, Springer-Verlag, 1960, 2, 625.

Huxley, H. E., Electron Microscopy, (Proc. Stockholm Conf. Electron Micr.), Stockholm, Almquvist, \& Wiksell, 1956, 260.
Huxuey, H. E., J. Biophysic. and Biochem. Cytol., $1957,3,631$.

Huxlex, H. E., Electron Microscopy, (Proc. 5th Internat. Congr. Electron Micr., Philadelphia), New York, Academic Press, 1962, 2, 0-1.

Kerridge, D., Horne, R. W., and Glauert, A. M., J. Mol. Biol., 1962, 4, 227.

Manton, I., and Clarke, B., J. Exp. Bot., 1952, 3, 265.

Roth, L. E., and Jenkins, R. A. Electron Microscopy, (Proc. 5th Internat. Congr. Electron Micr., Philadelphia), New York, Academic Press, 1962, 2, NN-3.

Schoenberg, C. F., Electron Microscopy, (Proc. 5th Internat. Congr., Electron Micr., Philadelphia), New York, Academic Press, 1962, 2, M-8. 